\title{
Celos, desesperanza e ideación suicida en población con orientación sexual diversa*
}

\section{Jealousy, Hopelessness and Suicidal Ideation in a Population with} Diverse Sexual Orientation

Recepción: 19 Abril 2018 | Aceptación: 03 Noviembre 2019

\author{
Bertha Lucía Avendaño-Prieto ${ }^{a}$ \\ Universidad Católica de Colombia, Colombia \\ ORCID: http://orcid.org/0000-0002-8136-5380 \\ Moisés Betancort Montesinos \\ Universidad de la Laguna, España \\ ORCID: http://orcid.org/0000-0002-7790-8557 \\ Angela Bernal-Aguirre \\ Universidad Católica de Colombia, Colombia \\ ORCID: http://orcid.org/0000-0003-0969-4507 \\ Laura Andrea González-Martínez \\ Universidad Católica de Colombia, Colombia \\ ORCID: http://orcid.org/0000-0003-0955-5304 \\ Stephany M. Gómez-SÁnChez \\ Universidad Católica de Colombia, Colombia \\ ORCID: http://orcid.org/0000-0002-8217-2185 \\ Christian F. Villalobos-Sánchez \\ Universidad Católica de Colombia, Colombia \\ ORCID: http://orcid.org/0000-0003-1471-5122
}

a Autor de correspondencia. Correo electrónico: blavendano@ucatolica.edu.co

Para citar este artículo: Avendaño-Prieto, B. L., Betancort Montesinos, M., Bernal-Aguirre, A., González-Martínez, L. A., Gómez-Sánchez, S. M., \& Villalobos-Sánchez, C. F. (2019). Celos, desesperanza e ideación suicida en población con orientación sexual diversa. Universitas Psychologica, 18(4), 1-12. https: //doi.org/10.11144/Javeriana.upsy18-4.cdis

\section{RESUMEN}

Los celos, la desesperanza y la ideación suicida son emociones que disminuyen la calidad de vida de quienes los padecen y a quienes afectan. En el imaginario social, prevalece la idea de que tales problemas se exacerban en las parejas constituidas por personas con orientación sexual diversa, razón por la cual se analizó la relación entre estas variables, en un grupo constituido por heterosexuales $(79 \%)$ y no heterosexuales (21 $\%)$. La muestra fue intencional, los participantes fueron 288 colombianos, mayores de 18 años. La estrategia metodológica utilizada fue predictiva transversal. Los instrumentos empleados fueron el CECLA que evalúa clases de celos en población colombiana y el Inventario de Desesperanza e Ideación Suicida (IDIS). Respecto a la relación entre la orientación sexual y la ideación suicida, los resultados mostraron diferencias entre los heterosexuales y los otros dos grupos (homosexuales y bisexuales) $F=10.49, p<0.05$. La prueba post hoc indicó que las diferencias se presentaron entre heterosexuales y homosexuales $p<0.05$ y entre heterosexuales y bisexuales $p<0$. No se encontraron diferencias estadísticamente significativas entre la orientación sexual y las variables celos y desesperanza. Pero independientemente de la orientación sexual, se halló relación entre la triada celos-desesperanza-ideación suicida, la cual se presenta en un modelo de ecuaciones estructurales.

Palabras clave

celos; ideación suicida; desesperanza; orientación sexual diversa; ecuaciones estructurales. 


\begin{abstract}
Jealousy, hopelessness, and suicidal ideation are emotions that diminish the quality of life of those who suffer and are affected from them. As in the social imaginary prevails the idea that such problems are exacerbated in couples consisting of people with diverse sexual orientation, the relationship between these variables in a group composed of heterosexuals (79\%) and non-heterosexuals (21\%) was analyzed. The sample was composed of 288 Colombian participants over 18 years old. The methodological strategy used was transversal predictive. The instruments used were the CECLA, that evaluates jealousy classes in the Colombian population, and the hopelessness and suicide ideation inventory (IDIS). Regarding the relationship between sexual orientation and suicidal ideation, the results showed differences between heterosexuals and the other two groups (homosexual and bisexual) $F$ $=10.49, p<0.05$. The post hoc test indicated differences between heterosexual and homosexual $p$ $<0.05$, and between heterosexual and bisexual $p<$ 0 . No statistically significant differences were found between sexual orientation and the variables jealousy and hopelessness. However, independently of the sexual orientation, a relationship was found between the triad jealousy - hopelessness - suicidal ideation, which is presented in a model of structural equations.

Keywords

jealousy; suicidal ideation; hopelessness; diverse sexual orientation; structural equations.
\end{abstract}

La orientación sexual es un concepto complejo cuyas formas cambian con el tiempo y difiere entre las culturas, es independiente del sexo biológico o de la identidad de género; se refiere a la capacidad de cada persona de sentir atracción emocional, afectiva y sexual y de mantener relaciones íntimas y sexuales con personas de un género diferente al suyo (heterosexualidad), de su mismo género (homosexualidad) o de más de un género (bisexualidad). (Oficina del Alto Comisionado para los Derechos Humanos [ACNUDH], 2013).

Las personas con orientación sexual no heterosexual sufren diferente tipo de discriminación y segregación. Respecto al análisis de las condiciones y calidad de trabajo, por ejemplo, el mercado laboral para este grupo no se caracteriza por ofrecer labores dignas que contribuyan al mejoramiento de su calidad de vida y a su realización plena bajo la perspectiva de justicia social e igualdad, partiendo del enfoque de capacidades, lo cual indica que estas personas se encuentran en situación de vulnerabilidad (Jiménez-Castaño, Cardona-Acevedo, \& Sánchez-Muñoz, 2017). Según la oficina del Alto Comisionado de las Naciones Unidas para los Refugiados (ACNUR), se ha documentado que las personas que pertenecen a alguno de los siguientes grupos: lesbiana, gay, bisexual, transgénero o intersex son blanco de homicidios, violencia sexual y de género, agresiones físicas, maltrato y tortura, detenciones arbitrarias, acusaciones de conducta inmoral, "desviada" o "antinatural", entre otros. Igualmente, dichas personas son discriminadas en el acceso a derechos sociales como el derecho al empleo, al más alto nivel posible de salud y a la educación (ACNUR, 2014).

Según Carroll y Mendos (2017), en el mundo, 72 Estados criminalizan las relaciones sexuales consensuales entre personas adultas del mismo sexo y la pena de muerte está "permitida", o existe evidencia de su existencia en ocho de estos. Por el contrario, en 22 Estados se reconocen y celebran matrimonios entre personas del mismo sexo. En Colombia, en el año 2009, la Corte Constitucional, igualó los derechos y deberes civiles, políticos y sociales de las parejas del mismo sexo con los de las parejas heterosexuales, a través de la Sentencia C-029/09. En el año 2016, se aprobó la Sentencia SU214/16, que incluyó la posibilidad de contraer matrimonio entre personas del mismo sexo, permitiendo con esto que las parejas con orientación sexual diversa puedan convivir como un matrimonio civil, situación que armoniza los principios de la dignidad humana, la libertad individual y la igualdad en materia de matrimonio en parejas homosexuales.

Aunque las parejas con orientación sexual diversa han venido conquistando sus derechos, la mayoría de las investigaciones realizadas sobre relaciones de pareja y el análisis del comportamiento de las personas en diferentes variables, como los celos, la desesperanza, la ideación suicida y demás constructos de interés para la psicología, se han realizado con personas o parejas heterosexuales. 
Los celos se han definido como "un sentimiento o emoción que surge como consecuencia de un exagerado afán de poseer algo de forma exclusiva (me perteneces...), su base es la infidelidad \# real o imaginaria\# de la persona amada" (Echeburúa \& FernándezMontalvo, 2001, p. 15). También se entienden como una respuesta emocional específica de rechazo social percibido, en la que se cree que un rival o un tercero se favorecerán por los recursos considerados beneficiosos para sí mismo. Los celos involucran sentir ira cuando hay pensamientos dirigidos a la infidelidad o a la deslealtad en la relación, que amenazan el vínculo con la pareja, ocasionando un conflicto interpersonal, humillación, incertidumbre y desesperanza. Los celos románticos a nivel patológico generan altos niveles de violencia (López-Ossorio, González Álvarez, BuquerínPascual, García-Rodríguez, \& Buela-Casal, 2017). Si el celoso pierde la relación de pareja, podría desarrollarse una depresión, por esto las situaciones de celos muy prolongadas en una relación de pareja generan sentimientos de desesperanza, que con frecuencia terminan en suicidios consumados (Reidl, 2005).

Bevan y Lanutti (2002) realizaron un estudio con 136 personas, 76 heterosexuales y 57 con identidad sexual diversa, también conocida como LGBTI (lesbiana, gay, bisexual, transexuales e intersexual). La investigación evaluó, en un primer momento, la experiencia de celos, diferenciándolos entre cognitivos o emocionales; posteriormente, midieron la expresión de celos por medio de respuestas comunicativas como amenazas, emociones negativas y manipulación, entre otras. Los resultados arrojados permitieron concluir que la orientación sexual y el sexo no tienen influencia directa en el nivel de intensidad en la expresión de celos cognitivos o emocionales o en las respuestas comunicativas que se empleen. Contrariamente, Frederick y Fales (2016), en un estudio realizado es los Estados Unidos con una muestra de 63894 participantes que incluía gay, lesbianas, bisexuales y heterosexuales, encontraron evidencias para concluir que las diferencias en los celos son más evidentes entre hombres y mujeres heterosexuales que entre hombres y mujeres homosexuales.

Calderón-Pérez, Flores-Galaz y Rivera-Aragón (2018), en una investigación sobre celos e infidelidad en personas heterosexuales y homosexuales, encontraron que los hombres suelen ser infieles sexualmente, independientemente de su orientación sexual, mientras que las mujeres homosexuales o heterosexuales, son infieles, sexual y emocionalmente, cuando hay una transgresión en la relación.

Acerca de los sentimientos que provocan celos, Monclús (2005) afirma que el sufrimiento percibido tanto por el sujeto celoso como por el sujeto celado, ocasiona una desestructuración total de la relación de la pareja, ya que cada uno de sus miembros vive simultáneamente emociones contradictorias, pasando del amor al odio o de la confianza a la desconfianza, lo que genera situaciones problemáticas que ocasionalmente inducen a conductas auto lesivas, como la ideación o el suicidio. Sobre este tema, en Estados Unidos, Australia y Reino Unido se ha encontrado que los adolescentes que se identifican como parte de la comunidad con orientación sexual diversa, presentan un alto índice en el padecimiento de trastornos mentales en relación con sus pares heterosexuales, de igual manera, el riesgo de presentar ideación e intentos de suicidio resulta alto en hombres gais y bisexuales (King et al., 2008). Así mismo, Zhao, Montoro, Igartua y Thombs (2010) reportaron resultados significativos en las tasas de suicidio en jóvenes con orientación sexual diversa, comparando los porcentajes con jóvenes heterosexuales.

El informe RaRE (Risk and resilience explored) elaborado por la Guía Vice de Salud Mental (2015), resultado de una colaboración entre la institución benéfica británica para la salud mental del colectivo de lesbianas, gais, bisexuales y transexuales (PACE) y tres universidades del Reino Unido, analizó los factores de riesgo y resiliencia para algunos problemas que afectan la salud mental del colectivo de lesbianas, gais, bisexuales y transexuales, entre estos, los intentos de suicidio 
en jóvenes LGBT menores de 26 años. Los datos fueron recolectados entre 2011 y 2014 , a través de dos series de entrevistas con 58 personas en total y una encuesta nacional de 2078 personas, en Inglaterra. Los resultados revelaron que un $34 \%$ de las lesbianas, gais y bisexuales jóvenes (menores de 26 años) encuestados, se había intentado suicidar por lo menos una vez en la vida. Además, el 48 \% de los transexuales jóvenes han intentado suicidarse. Las principales causas identificadas fueron la intimidación homofóbica o transfóbica y problemas por ser lesbiana, gay, bisexual o transexual en los entornos familiar y educativo.

En esta misma línea, Nystedt, Rosvall y Lindström (2019) en Suecia, realizaron un estudio transversal cuyo objetivo era investigar las asociaciones entre la orientación sexual y la experiencia de pensamientos suicidas e intentos de suicidio donde participaron 28029 personas entre 18 y 80 años. Se encontró que los hombres bisexuales y homosexuales y las mujeres bisexuales tenían una probabilidad significativamente más alta de pensamientos suicidas y tasas de probabilidad de intento de suicidio también significativamente más altas que los hombres y mujeres heterosexuales.

A pesar de lo mencionado con anterioridad, no se puede inferir que las personas con orientación sexual diversa sean más propensas que los heterosexuales a desarrollar un trastorno, o que la homosexualidad, por ejemplo, sea causa de mayores niveles de riesgo suicida; lo que puede entrar en consideración es el rol que juega el contexto como factor que ejerce presión hacia dicho sector de la población, subvalorando las condiciones sexuales con respecto a los heterosexuales. Lo anterior muestra que, desde un contexto social, el sector con orientación diversa no se encuentra en igualdad de condiciones respecto a un goce de derechos, en comparación con parejas heterosexuales; debido a esto suelen presentarse sentimientos de inadecuación que tienden a afectar su autoestima, ocasionando victimizaciones que a su vez conducen a estados de depresión con síntomas de desesperanza, una vía que aumenta los índices de suicidio o riesgo suicida (Pineda, 2013).

La ideación, el intento y la conducta suicida conforman la ruta del suicidio; se entiende la ideación suicida como un conjunto de pensamientos enfocados hacia la pérdida de la vida y conductas que ocasionen autodestrucción (Ceballos-Ospino et al., 2015). La intención suicida, a su vez, es considerada como todo deseo e intención de realizar un acto autodestructivo que lleve a la muerte e incluye de manera explícita la ideación suicida. El intento suicida se relaciona con toda acción autoinfligida, con el propósito de hacerse daño de manera letal y muchas veces es considerado conducta suicida no fatal (World Health Organization [WHO], 2012). La conducta suicida se define como "toda acción producida con el propósito explícito o implícito de quitarse la vida, incluyendo también cualquier intención, ideación o pensamiento que puede finalizar en dicha acción, aun cuando no hubiere ninguna expresión manifiesta" (Secretaría de Determinantes de la Salud y Relaciones Sanitarias \& Ministerio de la Salud de la Nación, 2012, p. 43).

La Organización Mundial de la Salud (OMS) afirma que el suicidio es un problema de salud pública mundial, y es una de las principales causas de muerte; los datos identifican que cerca de 800 000 personas se suicidan cada año y un intento de suicidio no consumado es el factor individual de riesgo más importante; además, el suicidio es la segunda causa principal de defunción en el grupo de 15 a 29 años de edad, el $78 \%$ de todos los suicidios se produce en países de ingresos bajos y medianos y la ingestión de plaguicidas, el ahorcamiento y las armas de fuego son algunos de los métodos más comunes de suicidio en todo el mundo (OMS, 2017).

En Colombia el comportamiento suicida también se considera un problema de salud pública y el suicidio se encuentra entre las tres primeras causas de muerte en las personas de 15 a 44 años. En la ciudad de Bogotá, en el año 2013, se encontró que la razón de suicidio con más prevalencia era el conflicto de pareja por factores como celos y desconfianza, con un porcentaje 
del $30.12 \%$ en la población (Cifuentes, 2013). Además, se presentó "un aumento progresivo de la tasa de suicidio en los últimos 10 años, pasando de 4.5 por cada 100000 habitantes para el año 2009 a 5.93 en el año 2018" (Instituto Nacional de Medicina Legal [INML], 2018).

Durante el año 2016, se reportaron 2310 suicidios, 242 casos (10.4 \%) más que en el año inmediatamente anterior. El decenio 2007-2016 acumuló 19177 casos, con una media de 1918 eventos por año, lo que indica 193 suicidios al mes y seis suicidios por día. La tasa de lesiones fatales autoinflingidas en 2016 fue de 5.2 eventos por cada 100000 habitantes. Además, al revisar el comportamiento de los factores que manifestaron las víctimas como desencadenantes de agresión por parte de su pareja, se encontró que el $36 \%$ de las mujeres y el $30 \%$ de los hombres manifestaron que por celos, desconfianza e infidelidad (INML, 2017).

Las variables de mayor relevancia para explicar los intentos suicidas son la desesperanza y la depresión, la primera más significativa que la segunda, puesto que la desesperanza como síntoma clínico de la depresión se asocia directamente con las conductas que buscan terminar con los vacíos existenciales percibidos (Beck, Steer, \& Brown, 1993). La desesperanza se define como "un gran sentimiento de pérdida de ánimo, la imposibilidad de poder imaginar que algo mejor pueda ocurrir, o el hecho de perder la confianza de que las cosas pueden cambiar y ser mejores" (Eguiluz, Rosales, \& Córdova, 2005, p. 312). Según García, Gallego y Pérez (2008), cuando las personas no alcanzan un logro existencial, se experimenta frustración asociada con desesperanza, la cual se caracteriza por dudas existenciales relacionadas con el sentido de la vida, este vacío existencial conlleva a estados de ánimo bajos, percepciones de falta de control sobre la propia vida y de metas a futuro. La desesperanza por causa existencial es un factor de riesgo para la presentación posterior de síntomas relacionados con el riesgo suicida.

Lo anterior lo corroboran Pérez, Marco y García-Alandete (2017), quienes, en un estudio realizado con 150 pacientes con un diagnóstico de trastorno mental, encontraron que la desesperanza afectiva se relaciona con los intentos suicidas; para estos autores, las autolesiones no suicidas y los intentos de suicidio se sitúan en el continuo de las conductas autolesivas, en el que los pacientes con una historia de intentos de suicidio constituyen el grupo de mayor gravedad.

Según estudios realizados en 2013 por la Federación Estatal de Lesbianas, Gais, Transexuales y Bisexuales (FELGTB), la desesperanza está relacionada con el acoso escolar por homofobia frecuente, y de manera prolongada puede provocar en los adolescentes lesbianas, bisexuales y gais, sentimientos de vulnerabilidad, aislamiento y culpabilidad, emociones cuya prolongación en el tiempo llevan a las víctimas con desesperanza a presentar pensamientos de no solución para la situación que se está sufriendo. En los estudios realizados por esta Federación se encontró alta correlación entre desesperanza y riesgo de ideación suicida.

Resultados similares reportó un estudio con una muestra de 67359 personas cuyo objetivo era examinar los patrones en la salud autoinformada por identidad de orientación sexual y género, las minorías sexuales (gais / lesbianas, $2 \%$ de muestra; bisexuales, $1 \%$ tenían mayor probabilidad de informar tensión o preocupación, consumo de cigarrillo, de drogas, victimización sexual de por vida y pruebas de VIH. En comparación con los heterosexuales, los bisexuales informaron sobre más barreras para la atención médica, tristeza actual, ideación suicida del año anterior y riesgo de enfermedad cardiovascular (Conron, Mimiaga, \& Landers, 2010).

Martínez-León, Peña, Salazar, García y Sierra (2017), realizaron una revisión sistemática de los celos románticos en investigaciones publicadas en las principales bases de datos y plataformas internacionales, hasta diciembre del 2016. Los 230 estudios seleccionados fueron clasificados en función de las variables asociadas con los celos: a) personales, entre las que se encuentra la orientación sexual, b) interpersonales que incluye la violencia y c) socioculturales. Los resultados evidencian asociación entre la violencia (física y verbal) y los celos. En el 41 
\% de los estudios no se informó la orientación sexual de los participantes, y respecto al suicidio, los autores mencionan la investigación realizada por Block \& Block (2012), quienes indican que el Chicago Homicide Dataset, reportó 1361 víctimas entre los años 1965 y 2000, donde los celos sexuales y la rivalidad sexual estaban presentes, y los delincuentes acusaron a sus víctimas de infidelidad. Después del asesinato, 275 perpetradores se suicidaron.

El interés principal para realizar este estudio sobre la relación existente entre celos, desesperanza e ideación suicida en población con orientación sexual diversa, se debe a las consecuencias que tiene cada una de las variables mencionadas en el comportamiento de los seres humanos. Los resultados ofrecerán información relevante para la psicología clínica en cuanto a diagnóstico, intervención y prevención de situaciones con un carácter potencialmente peligroso en las relaciones sociales. Teniendo en cuenta el anterior marco de referencia se planteó como objetivo general identificar la relación existente entre desesperanza, ideación suicida y celos, en población colombiana con orientación sexual diversa.

\section{Método}

\section{Tipo de estudio}

Se utilizó una estrategia predictiva transversal puesto que el objetivo fue explorar una relación funcional entre variables (Ato, López, \& Benavente, 2013).

\section{Participantes}

Para la selección de la muestra se tuvo en cuenta que los participantes fueran colombianos mayores de edad, que hubieran tenido al menos una relación sentimental en su vida. La muestra inicial estuvo conformada por 411 participantes, se eliminaron menores de edad y pruebas incompletas, quedando así una muestra de 288 participantes (62.8\% mujeres y $37.2 \%$ hombres),
$8.3 \%$ bisexuales, $12.8 \%$ homosexuales y $78.8 \%$ heterosexuales.

\section{Instrumentos}

Las variables sociodemográficas fueron evaluadas con preguntas elaboradas por los investigadores para el presente estudio. Las demás variables de interés se evaluaron con instrumentos diseñados con población colombiana los cuales se describen a continuación.

Inventario de Desesperanza e Ideación Suicida (IDIS)

Instrumento que busca identificar la frecuencia de cogniciones disfuncionales en la conducta suicida, como son Ideación Suicida (IS) y Desesperanza (DS), está compuesto por 11 ítems para la escala de Desesperanza y 10 ítems para Ideación Suicida. El índice de consistencia interna con el coeficiente alfa de Cronbach fue de $\alpha=0.77$ para la escala IS y de $\alpha=0.86$ para la escala de DS. Los ítems del IDIS se ajustaron a los parámetros del modelo de Rasch, de tal forma que el INFIT de los ítems de la escala IS osciló entre 0.75 y $1.22(\mathrm{M}=0.97, \mathrm{DE}=0.14)$ y el OUTFIT entre 0.66 y $1.14(\mathrm{M}=0.92, \mathrm{DE}=$ 0.16), para la escala DS, el INFIT osciló entre 0.85 y $1.3(\mathrm{M}=1.0 \mathrm{DE}=0.15)$ y el OUTFIT entre 0.78 y $1.33(M=0.99, D E=0.18)$. Las correlaciones biseriales estuvieron por encima de 0.25 (Toro, Avendaño, \& Castrillón, 2016).

\section{CECLA}

Instrumento diseñado para evaluar clases de celos patológicos en población colombiana, consta de 19 ítems (siete evalúan celos pasionales, cuatro celos obsesivos y ocho celos delirantes). Los valores encontrados por los autores en el coeficiente $r$ de Pearson utilizado para establecer la correlación test-retest fue de 0.94. Los valores del alfa de Cronbach para las subescalas fueron los siguientes: factor celos delirantes $\alpha=0.89$, factor celos obsesivos $\alpha$ 
$=0.87$ y factor celos pasionales $\alpha=0.84 \mathrm{La}$ fiabilidad Omega alcanzó un valor de 0.94. Los ítems del CECLA se ajustaron a los parámetros del modelo de Rasch, los valores INFIT y OUTFIT, en el grupo de los celos delirantes oscilaron entre 0.78 y 1.29 , para el grupo de ítems que evalúa celos obsesivos estos valores se encontraron entre 0.68 y 0.92 y para el grupo de ítems que evalúa celos pasionales estos valores oscilaron entre 0.75 y 1.29 , indicadores que muestran ajuste al modelo (Avendaño, Betancort, \& Peñate, 2016).

\section{Análisis de datos}

Inicialmente, se realizaron los análisis descriptivos de las variables sociodemográficas y para establecer la relación entre las variables celos, desesperanza e ideación suicida con la variable orientación sexual diversa, se realizaron tres ANOVAS de una vía. Se utilizó el paquete estadístico SPSS V25 para los análisis univariados y bivariados y el IBM SPSS Amos para las ecuaciones estructurales.

\section{Consideraciones éticas}

Se respetaron las normas éticas y legales de la investigación según la legislación nacional e internacional, particularmente, lo establecido por el Ministerio de Salud (1993) en la Resolución 8430, según la cual este estudio se cataloga como riesgo inferior al mínimo, atendiendo los principios éticos de confidencialidad, reserva de información, no maleficencia y beneficio para la ciencia.

\section{Resultados}

Los datos generales de la población que participó en la investigación permiten señalar que la edad promedio de los 288 participantes fue de 31 años, con un mínimo de 18 y un máximo de 75 años. Los participantes eran oriundos de la región Amazónica (1\%), Andina (84\%), Caribe (4.9 $\%)$, Insular (3.8 \%), Orinoquia (2.8 \%) y Pacífica
(3.5\%). Todos los niveles socioeconómicos estuvieron representados: el $21.6 \%$ pertenecían al nivel bajo, el $72.9 \%$ al medio y el porcentaje restante $(5.6 \%)$ al nivel alto. En cuanto al nivel de educación, el $0.3 \%$ contaba con educación básica primaria, el $12.5 \%$ con educación media y el $87.2 \%$ con educación superior. Sobre el estado civil, el $31.3 \%$ de la población estaba casada, el $4.2 \%$ separada o divorciada, el $35.1 \%$ se encontraba soltero con pareja actual, el $28.8 \%$ estaba soltero sin pareja actual y el $0.7 \%$ restante era viudo; aunque el $33.7 \%$ al momento del estudio no tenía pareja estable, cumplía con el criterio de inclusión de haber tenido al menos una relación sentimental en su vida, y el tiempo de relación con la pareja osciló entre un mínimo de un mes y un máximo de cincuenta años, con un promedio de cinco años y medio.

En cuanto a la ocupación actual, se encontraban desempleados (7.3\%), empleados (37.8\%), estudiantes (41\%), independientes $(12.8 \%)$ y pensionados (1 \%). El $71 \%$ refirió haber sido infiel alguna vez, y el $56 \%$ se considera celoso. La correlación entre las variables edad y tiempo de relación en pareja, aunque significativa, fue baja $(r=-0.15, p<0.05$ y $r=-0.12, p<0.05)$.

Para analizar la relación entre la orientación sexual y las variables celos, desesperanza e ideación suicida, se realizaron tres análisis de varianza de una vía. En cuanto a los celos, se halló que, aunque la media y la desviación estándar del grupo de bisexuales fueron superiores a las de los otros dos grupos, no se encontraron diferencias estadísticamente significativas $(F=1.57, p=$ 0.21). Las medias y desviaciones estándar de los tres grupos fueron las siguientes: heterosexuales $(M=32.49, D E=11.70)$, homosexuales $(M=$ $32.57, D E=14.33)$ y bisexuales $(M=37.21, D E$ $=15.94)$.

Con respecto a la relación entre la orientación sexual y la ideación suicida, los resultados mostraron diferencias entre los heterosexuales y los otros dos grupos (homosexuales y bisexuales) $F=10.49, p<0.05$. Las medias y desviaciones estándar de los tres grupos fueron las siguientes: heterosexuales $(\mathrm{M}=1.07, \mathrm{DE}=1.91)$, homosexuales $(M=2.05, D E=2.64) \mathrm{y}$ 
bisexuales $(M=2.83, D E=2.50)$. La prueba post hoc indicó que las diferencias se presentaron entre heterosexuales y homosexuales $p<0.05$ y entre heterosexuales y bisexuales $p<0$. No se encontró diferencia entre homosexuales y bisexuales $(p=0.356)$. No se encontró asociación significativa entre la orientación sexual (bisexual, homosexual y heterosexual), con la desesperanza $(F=0.32, p=0.73)$.

Independientemente de la orientación sexual, se analizó la posible relación entre los celos, la desesperanza y la ideación suicida. La Tabla 1 muestra los índices de ajuste de los tres modelos analizados. El modelo uno (M1) se realizó relacionando directamente los celos y la desesperanza con la ideación suicida, sin establecer asociación entre las dos primeras. El modelo dos (M2) se desarrolló relacionando directamente los celos y desesperanza con ideación suicida, pero estableciendo asociación entre las dos primeras. El modelo tres (M3), que representa la relación entre las variables celos e ideación suicida mediadas por la desesperanza, presentó el mejor ajuste. La estructura general propuesta y los resultados estimados entre las variables observadas y las variables latentes, muestran que los celos tienen un efecto sobre la desesperanza y a su vez la desesperanza se relaciona con la ideación suicida (Figura 1).

Tabla 1

Índices de bondad de ajuste de los tres modelos analizados entre celos, DS e IS

\begin{tabular}{lrllllrll}
\hline M & CMIN/DF & GFI & IFI & TLI & CFI & RMSEA & LO & HI \\
\hline M1 & 2.63 & 0.84 & 0.89 & 0.88 & 0.89 & 0.08 & 0.07 & 0.08 \\
M2 & 4.44 & 0.89 & 0.86 & 0.81 & 0.86 & 0.11 & 0.09 & 0.13 \\
M3 & 1.79 & 0.90 & 0.95 & 0.94 & 0.95 & 0.05 & 0.04 & 0.06 \\
\hline
\end{tabular}

Figura 1

Modelo de mejor ajuste entre las variables analizadas. (M3)

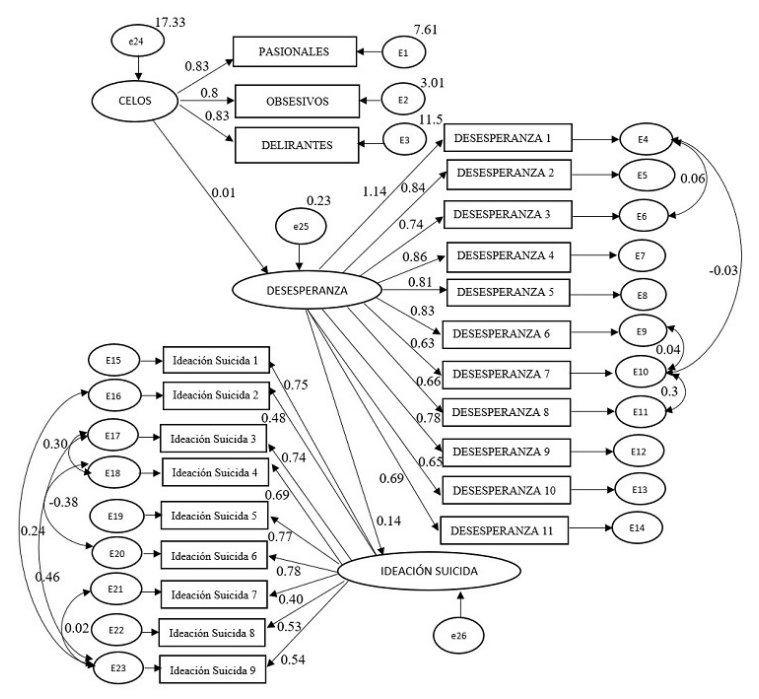

Los índices de bondad de ajuste obtenidos con el AMOS, fueron: la razón entre el valor de ji-cuadrado $\left(\chi^{2}\right)$ y el número de grados de libertad correspondientes $\chi 2 / g l$ cuya puntuación fue $=1.79$, valores inferiores a tres indican un buen ajuste. El GFI $=0.9$, índice global de ajuste (Goodness of Fit Index) y el CFI = 0.95, índice de ajuste comparativo (Comparative Fit Index), varían entre 0 y 1 , donde 0 indica ausencia de ajuste y 1 ajuste óptimo, valores de 0.95 o superiores son considerados excelentes (Pilatti, Godoy, \& Brussino, 2012). El RMSEA $=0.05$, error cuadrado de aproximación a las raíces medias (Root Mean Square Error of Approximation), el cual es considerado óptimo cuando sus valores son de 0.05 o inferiores y aceptables en el rango [0.05; 0.08] $(\mathrm{Hu} \&$ Bentler, 1998). El intervalo de confianza del RMSEA osciló entre [0.05 y 0.06].

\section{Discusión}

El objetivo de la presente investigación fue identificar la relación existente entre ideación suicida, desesperanza y celos en población colombiana con orientación sexual diversa. Los hallazgos confirman los resultados presentados por King et al. (2008) en Estados Unidos, 
Australia y Reino Unido, Zhao et al. (2010) y Nystedt et al. (2019) en Suecia, quienes encontraron riesgo de presentar ideación e intentos de suicidio en hombres gay y bisexuales en relación con sus pares heterosexuales.

No se encontró relación entre los celos con la orientación sexual, lo que corrobora el estudio de Bevan y Lanutti (2002), quienes concluyeron que la orientación sexual no tiene incidencia en el nivel de expresión de celos. Asimismo, los resultados presentados por Frederick y Fales (2016) mostraron que las diferencias existen solamente para individuos heterosexuales, hombres y mujeres bisexuales no presentan diferencias significativas entre ellos con respecto a la infidelidad sexual (30 \% vs. 27 $\%)$, sin importar si su pareja era hombre (35\% vs. $29 \%$ ) o mujer (28 \% vs. $20 \%$ ), hombres gay y lesbianas, tampoco presentaron diferencias (32\% vs. $34 \%)$.

Contrario a los resultados de 2013 presentados por la Federación Estatal de Lesbianas, Gais, Transexuales y Bisexuales (FELGTB), y a los hallazgos presentados por Conron et al. (2010), en el presente estudio no se halló relación entre la desesperanza y la orientación sexual.

Sin embargo, al analizar los datos de la muestra total sin diferenciar la orientación sexual, se encontró que los celos sí tienen un efecto sobre la desesperanza, que a su vez se encuentra relacionada con la ideación suicida, resultados que apoyan las afirmaciones de Monclús (2015), quien refiere que los celos producen efectos relacionados con conductas autolesivas, ideación y actos suicidas, fundamentados en el amor, el odio y la desconfianza. Esta relación también la manifestó Reidl (2005), quien expuso que los celos prolongados en una relación de pareja generan sentimientos de desesperanza y finalizan con el suicidio.

Se confirma también lo expuesto por Cifuentes (2013), quien afirmó que en Bogotá una de las causas con mayor prevalencia $(30.12 \%)$ del suicidio, está relacionada con el conflicto de pareja por factores como: celos, infidelidad y desconfianza. Los hallazgos del presente estudio ofrecen evidencia de la relación entre la triada: celos, desesperanza e ideación suicida, como elementos que se deben tener en cuenta en la intervención clínica, con el objetivo de disminuir el riesgo de suicidio que ocasionan los celos. Detectar la presencia de desesperanza e ideación suicida en los contextos académico, social y familiar, evitará la destrucción de muchos proyectos de vida (Quintanilla, Haro, Flores, Celis de la Rosa, \& Valencia, 2003).

El estudio realizado permite afirmar que los celos y la desesperanza son indicadores de riesgo de ideación suicida. Por lo tanto, sería apropiado relacionar estas variables en estudios con muestras representativas de la población. Si a lo anterior se suma que los estereotipos relacionados con las personas que poseen orientación sexual diversa pueden tener consecuencias negativas en ellos, se resalta la necesidad de realizar futuros estudios con esta población.

La distribución porcentual de los participantes no fue equivalente, el porcentaje de participación de personas con orientación sexual diversa fue $21 \%$, sin embargo, este indicador es superior al informado a nivel nacional en la última Encuesta Nacional de Demografía y Salud (ENDS), en la cual se reporta que "el 99 por ciento de las mujeres y el 98.2 por ciento de los hombres entrevistados son heterosexuales, el 0.4 por ciento de las mujeres y el 1.2 por ciento de los hombres son homosexuales y, el 0.6 por ciento de las mujeres y el mismo porcentaje de los hombres son bisexuales" (Ministerio de Salud y Protección Social, 2015, p. 44). Aun así, los resultados no se pueden generalizar a la población colombiana con esta orientación, puesto que la muestra no fue seleccionada al azar, todos los participantes fueron voluntarios y conocían el objetivo del estudio.

\section{Referencias}

Ato, M., López, J. J., \& Benavente, A. (2013). Un sistema de clasificación de los diseños de investigación en psicología. Anales de Psicología, 29(3), 1038-1059. https://doi.org /10.6018/analesps.29.3.178511 
Avendaño, B. L., Betancort, M., \& Peñate, W. (2016). Diseño de un instrumento para evaluar los celos. Aproximación a un perfil socio-demográfico y psicológico de la conducta celotípica (Disertación doctoral no publicada). Universidad de la Laguna, Tenerife, España.

Beck, A. T., Steer, R., \& Brown, G. (1993). Dysfunctional attitudes and suicidal ideation in psychiatric outpatients. Suicide Life-Threatening Behavior, 23(4), 11-20. https://doi.org/10.1111/j.1943-278X .1993.tb00274.x

Bevan, J., \& Lanutti, P. (2002). The experience and expression of romantic jealousy in same-sex and oppositesex romantic relationships. Communication Research Reports, 19(3), 258-268. https://do i.org/10.1080/08824090209384854

Block, C.R., \& Block, R. (2012). Margo Wilson 's Contributions to the Chicago Homicide Dataset: Sexual rivalry and sexual jealousy. Homicide Studies, 16(4), 404-427. https://d oi.org/10.1177/1088767912461142

Calderón-Pérez, Y., Flores-Galaz, M. M., \& Rivera-Aragón, S. (2018). Celos e infidelidad en personas heterosexuales y homosexuales: estudio intracultural. Acta de Investigación Psicológica, 8(1), 17-28. https://doi.org/10.22201/fpsi.20074 719e.2018.1.02

Carroll, A., \& Mendos, L. R. (2017). Homofobia de Estado. Estudio jurídico mundial sobre la orientación sexual en el derecho: criminalización, protección y reconocimiento (12. ${ }^{\text {a }}$ ed.). Ginebra: Asociación Internacional de Lesbianas, Gays, Bisexuales, Trans e Intersex.

Ceballos-Ospina, G., Suárez-Colorado, Y., Suescún-Arregocés, J., Gamarra-Vega, L., González, K., \& Sotelo-Manjarres A. (2015). Ideación suicida, depresión y autoestima en adolescentes escolares de Santa Marta. Revista Internacional de Ciencias de la Salud Duazary, 12 (1), 15-22.h ttps://doi.org/10.21676/2389783X.1394

Conron, K. J., Mimiaga, M. J., \& Landers, S. J. (2010). A population-based study of sexual orientation identity and gender differences in adult health. American Journal of Public Health, 100(1),1953-1960. Recuperado de https://www.scopus.com/record/display. uri? eid $=2$-s2.0-77956601963 \&origin $=$ inw ard\&txGid =b8b75379adf40e913ebbc31d 66f741ea

Cifuentes, S. L. (2013). Comportamiento del suicidio. Bogotá: Instituto Nacional de Medicina Legal y Ciencias Forenses. Recuperado de http://www.medicinalegal.gov.co/docu ments/10180/188820/FORENSIS+2013+ 3-+ suicidio.pdf/65a683b4-38b2-46a4-b32 $\mathrm{a}-\mathrm{f} 2 \mathrm{a} 088 \mathrm{~b} \mathrm{~b} 25 \mathrm{bf}$

Corte Constitucional de Colombia. Sentencia C-029 de 2009 (M. P. Rodrigo Escobar Gil: 28 de enero de 2009). Recuperado de http://www.corteconstitucional.gov.co/r elatoria/2009/c-029-09.htm

Corte Constitucional de Colombia. Sentencia de unificación SU214 de 2016 (M. P. Alberto Rojas Ríos: 28 de abril de 2016). Recuperado de http://www.corteconstituci onal.gov.co/relatoria/2016/su214-16.htm

Echeburúa, E., \& Fernández-Montalvo, J. (2001). Celos en la pareja: una emoción destructiva. Barcelona: Ariel.

Eguiluz, L., Rosales, J., \& Córdova, M. (2005). La didáctica constructiva de una escala de desesperanza: resultados preliminares. Enseñanza e Investigación en Psicología, 10(2), 311-324. Recuperado de http://www .redalyc.org/articulo.oa?id $=29210207$

Federación Estatal de Lesbianas, Gais, Transexuales y Bisexuales. (2013). Acoso escolar (y riesgo de suicidio) por orientación sexual e identidad de género: fracaso del sistema educativo (Informe para el Defensor del Pueblo). Madrid: Autor. Recuperado de http://www.felgtb.org/rs/2157/d112d6ad -54ec-438b-9358-4483f9e98868/671/filena me/informedefensor-17m13.pdf

Frederick, D. A., \& Fales, M. R. (2016). Upset over sexual versus emotional infidelity among gay, lesbian, bisexual, and heterosexual adults. Archives of Sexual 
Behavior, 45(1), 175-191. https://doi.org/10 .1007/s10508-014-0409-9

García, J., Gallego, J., Pérez, E. (2008). Sentido de la vida y desesperanza: un estudio empírico. Universitas Psychologica, 8(2), 447-454. Recuperado de http://www.scielo. org.co/pdf/rups/v8n2/v8n2a12.pdf

Guía Vice de Salud Mental. (2015). La salud mental LGBT: iestamos haciendo lo suficiente? Recuperado de https://www.vice.com/es_co/article/nng nzm/la-salud-mental-lgbt-estamos-haciend o-lo-suficiente-877

Hu, L., \& Bentler, P. M. (1998). Fit indices in covariance structure modeling: Sensitivity to underparameterized model misspecification. Psychological Methods, 3(4), 424-453. Recuperado de http://people.cehd.tamu.edu/ okwok/e psy651R/Articles/HuBentler1998.pdf

Instituto Nacional de Medicina Legal. (2017). Boletín de Prensa. Forensis Datos para la vida. 2016. Recuperado de http://www.medicinalegal.gov.co/docu ments/20143/49526/Forensis +2016.+Dat os + para + la + vida.pdf

Instituto Nacional de Medicina Legal. (2018). Boletín de Prensa. Forensis Datos para la vida. 2018. Recuperado de http://www.medicinalegal.gov.co/docu ments/20143/386932/Forensis +2018.pdf/b e4816a4-3da3-1ff0-2779-e7b5e3962d60

Jiménez-Castaño, J., Cardona-Acevedo, M., \& Sánchez-Muñoz, M. P. (2017). Discriminación y exclusión laboral en la comunidad LGBT: un estudio de caso en la localidad de Chapinero, Bogotá, Colombia. Papeles de Población, 23(93). https://doi.org/ 10.22185/24487147.2017.93.028

King, M., Semlyen, J., Tai, S., Killaspy, H., Osborn, D., Popelyuk, D., \& Irwin, N. (2008). A systematic review of mental disorder, suicide and deliberate self-harm in lesbian, gay and bisexual people. BMC Psychiatry, 8(70), 1-17. https://doi.org/10.1 186/1471-244X-8-70

López-Ossorio, J. J., González-Álvarez, J. L., Buquerín-Pascual, S., García-Rodríguez, L.
F., \& Buela-Casal, G. (2017). Risk factors related to intimate partner violence police recidivism in Spain. International Journal of Clinical and Health Psychology, 17, 107-119. https://doi.org/10.1016/j.ijchp.2016.12.001

Resolución 8430 de 1993 [Ministerio de Salud Nacional]. Por la cual se establecen las normas científicas, técnicas y administrativas para la investigación en salud. Octubre 4 de 1993. Recuperado de ht tps://www.minsalud.gov.co/sites/rid/Lists/B ibliotecaDigital/RIDE/DE/DIJ/RESOLUCI ON-8430-DE-1993.PDF

Martínez-León, N. C., Peña, J. J., Salazar, H., García, A., \& Sierra, J. C. (2017). A systematic review of romantic jealousy in relationships. Terapia Psicológica, 35(2), 203-212. https://doi.org/10.4067/s0718-48 082017000200203

Ministerio de Salud y Protección Social. (2015). Encuesta Nacional de Demografía y Salud. Recuperado de https://dhsprogram.com/pu bs/pdf/FR334/FR334.pdf

Monclús, E. (2005). Celos, celos patológicos y delirio celotípico. Revista de Psiquiatría Facultad de Medicina de Barna, 32 (1), 14-22. Recuperado de https://dialnet.unirioja.es/se rvlet/articulo? codigo $=1165902$

Naciones Unidas. Derechos Humanos. Oficina del Alto Comisionado. (2013). Orientación sexual e identidad de género en el derecho internacional de los derechos humanos. Recuperado de http://acnudh.org/wp-conte nt/uploads/2013/11/orentaci\%C3\%B3n-se xual-e-identidad-de-g\%C3\%A9nero2.pdf

Nystedt, T., Rosvall, M., \& Lindström, M. (2019). Sexual orientation, suicide ideation and suicide attempt: A population-based study. Psychiatry Research, 275, 359-365. https://d oi.org/10.1016/j.psychres.2019.04.007

Oficina del Alto Comisionado de las Naciones Unidas para los Refugiados. (2014). La protección internacional de las personas LGBTI. Recuperado de https://www.acnur.org/fileadmin/Docu mentos/Publicaciones/2014/9872.pdf

Organización Mundial de la Salud. (2017). Datos y cifras sobre el suicidio infografía. 
Recuperado de http://www.who.int/mental _health/suicide-prevention/infographic/es/

Pérez, S., Marco, J. H., \& García-Alandete, J. (2017). The role of hopelessness and meaning in life in a clinical simple with nonsuicidal self-injury and suicide attempts. Psicothema, 29(3), 323-328. https://doi.org/ 10.7334/psicothema2016.284

Pilatti, A., Godoy, J. C., \& Brussino, S. A. (2012). Análisis factorial confirmatorio del cuestionario de expectativas hacia el alcohol para adolescentes (CEA-A). Acta Colombiana de Psicología, 15(2), 11-20. Recuperado de http://www.scielo.org.co/pd f/acp/v15n2/v15n2a02.pdf

Pineda, C. (2013). Etiología social del riesgo de suicidio en adolescentes y jóvenes lesbianas, gay y bisexuales: una revisión. Psicogente, 16(29), 218-234. Recuperado de http://revistas.unisimon.edu.co/index.p hp/psicogente/article/view/1953

Quintanilla Montoya, R., Haro Jiménez, L., Flores Villavicencio, M., Celis de la Rosa, A., \& Valencia Abundiz, S. (2003). Desesperanza y tentativa suicida. Investigación en Salud, 5(2). Recuperado de http://www.redalyc.org/articulo.oa?id= 14250206

Reidl, L. C. (2005). Celos y envidia: emociones humanas. México: Universidad Nacional Autónoma de México.

Secretaría de Determinantes de la Salud y Relaciones Sanitarias \& Ministerio de la Salud de la Nación (SDSRS-MS). (2012). Lineamientos para la Atención del Intento de Suicidio en Adolescentes.Buenos Aires, Argentina: DCV Anahí Maronas.

Toro, R., Avendaño-Prieto, B. L., \& Castrillón, D. A. (2016). Design and psychometric analysis of the Hopelessness and Suicide Ideation Inventory "IDIS". International Journal of Psychological Research, 9(1), 52-63. Recuperado de http://www.scielo.org.co/scielo.php?scri pt $=$ sci_arttext\&pid $=$ S2011-20842016000 100006

World Health Organization. (2012). For which strategies of suicide prevention is there evidence of effectiveness? Copenhagen, DK: WHO Regional Office for Europe.

Zhao, Y., Montoro, R., Igartua, K J., \& Thombs, B. D. (2010). Suicidal ideation and attempt among adolescents reporting "unsure" sexual identity or heterosexual identity plus same sex attraction or groups? Journal of the American Academy of Child $\mathbb{E}$ Adolescent Psychiatry, 49(2), 104-113. https ://doi.org/10.1016/j.jaac.2009.11.003

\section{Notas}

* Artículo de investigación. 